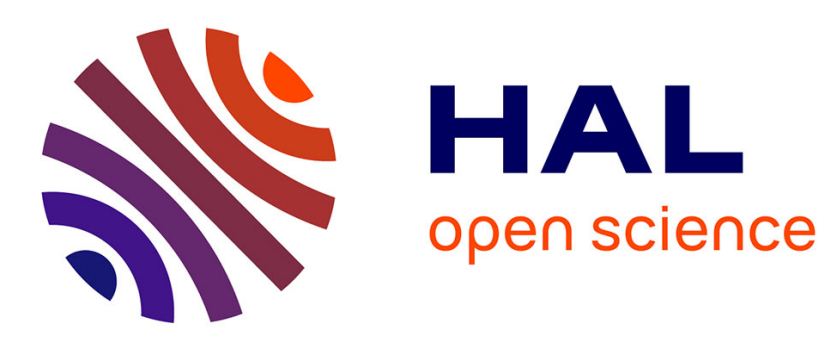

\title{
Esquisse d'une analyse des régulations à laquelle pourrait contribuer l'Economie des Conventions
}

Olivier Thevenon

\section{To cite this version:}

Olivier Thevenon. Esquisse d'une analyse des régulations à laquelle pourrait contribuer l'Economie des Conventions. L'Economie des Conventions: méthodes et résultats, La Découverte, pp.165-192, 2006, Essais et Recherches. hal-00417463

\section{HAL Id: hal-00417463 \\ https://hal.science/hal-00417463}

Submitted on 16 Sep 2009

HAL is a multi-disciplinary open access archive for the deposit and dissemination of scientific research documents, whether they are published or not. The documents may come from teaching and research institutions in France or abroad, or from public or private research centers.
L'archive ouverte pluridisciplinaire HAL, est destinée au dépôt et à la diffusion de documents scientifiques de niveau recherche, publiés ou non, émanant des établissements d'enseignement et de recherche français ou étrangers, des laboratoires publics ou privés. 


\title{
Chapitre 17
}

\section{Esquisse d'une analyse des régulations à laquelle pourrait contribuer l'Economie des Conventions}

\author{
Olivier Thévenon
}

La littérature qui s'est récemment développée autour de variété des capitalismes est issue de traditions d'analyse très diverses. Dans cet ensemble, la théorie de la régulation met en avant les modes de régulation variables qui «soutiennent» et «pilotent» le «régime d'accumulation en vigueur» dans les différents pays [Boyer, 2004, p. 20]. Le mode de régulation désigne alors « tout ensemble de procédures et de comportements, individuels et collectifs qui ont la propriété de reproduire les rapports sociaux fondamentaux à travers la conjonction de formes institutionnelles historiquement déterminées »; il assure en outre «la compatibilité dynamique d'un ensemble de décisions décentralisées, sans que soit nécessaire l'intériorisation par les acteurs économiques des principes de l'ajustement de l'ensemble du système » [idem, p. 20].

La forme variable que peut prendre le mode de régulation selon les pays dépend alors de la particularité des formes institutionnelles (et plus généralement aux institutions), qui désigne la codification particulière que reçoivent les «rapports sociaux fondamentaux » dans un contexte économique et historique donnés. Ces formes institutionnel- 
les sont alors le résultat de compromis « institutionnalisés » entre une logique économique et des choix politiques historiquement situés.

De là, l'approche en termes de régulation rejoint certaines perspectives « institutionnalistes » qui se distinguent de l'analyse économique mainstream dans la mesure où le rôle des institutions n'est plus seulement considéré à travers leur effet sur le système de prix (objectif ou implicite) associé aux différentes options de comportement, mais en amont par le fait qu'elles en médiatisent des cadres cognitifs - composés de symboles, de processus cognitifs et de valeurs - modelant les schémas d'interprétation et d'évaluation qui sous-tendent les pratiques et la perception des intérêts [Hall et Taylor, 1997]. Ces cadres cognitifs supportent des formes d'évaluation spécifiques qui induisent un formatage variable des institutions: il en résulte des régularités de comportement différentes et différentes manières dont les individus mobilisent ces institutions.

Une autre proximité peut être établie, cette fois ci avec l'approche sociétale qui met en avant les formes variables «d'encastrement» structurant les relations économiques au sein de codifications sociales et institutionnelles très différentes selon les pays [Théret, 1997]. Les interactions s'établissant entre les différentes sphères de rapports sociaux conduisent à envisager un modèle d'explication systémique, dont la cohérence peut être plus ou moins assurée par les institutions. Deux impératifs s'imposent toutefois pour analyser ces cohérences :

D'abord, il s'agit de ne pas négliger l'hétérogénéité des modes de régulation qui peuvent être observés dans un pays et un moment donnés De cette diversité dépend directement celle des choix et comportements possibles auxquels l'ensemble des institutions donne plus ou 
moins de possibilité d'être réalisés. Ce point fut au centre de la critique formulée par J.-D. Reynaud [1979] à l'encontre de l'approche sociétale qui met en avant le poids des « cohérences nationales » engendrées par des relations de fortes interdépendances [Maurice, 2000]. En acceptant la «fiction» du système, le risque est en effet d'introduire un biais d'observation dont le principal défaut est d'ignorer la pluralité des compromis et des régulations qui se forment, de façon locale, à l'opposé d'un postulat d'unité.

Il s'agit ensuite de pouvoir apprécier le degré proximité des comportements et des formes institutionnelles observées d'un pays à l'autre, sans postuler a priori de trop fortes similarités ou donner au contraire trop de poids aux différences. Certes, le problème est de reconnaître que «la spécificité véhicule une forme de généralité qui est diffusée à partir d'elle et cristallisée en elle » [Maurice et al., 1982, p. 314], mais pour le reconnaître, il faut disposer d'une théorisation qui soit suffisamment générale pour permettre d'identifier à la fois les points communs des formes de régulations au-delà de leur spécificité concrètes, et la distance qui sépare les différents pays. Cela implique la construction "d'idéaux-types » à caractère très général dans lequel chaque pays peut s'inscrire comme forme particulière [Théret, 1997; Berthelot, 2000].

L'argument central de cette contribution est que la pleine compréhension de la variété des modes de régulation (de la cohérence infranationale, et différences internationales qui en sont dérivées) nécessite de considérer les registres de justification qui sous-tendent et structurent les formes institutionnelles, et les inscrit dans une «dynamique». Il s'agit dès lors d'examiner les caractéristiques des régulations par- 
tielles qui peuvent s'établir pour chaque forme institutionnelle (ou à leur interface), et de considérer la cohérence de la dynamique d'ensemble que peuvent impulser ces dernières, via les institutions et les politiques publiques. On ne postule donc pas a priori l'existence d'une dynamique parfaitement stabilisée puisqu'il est concevable, comme le souligne Boyer [2004], que les comportements suscités par les diverses formes institutionnelles soient incompatibles et résultent en une dynamique incohérente [p. 20]. Pour caractériser ces régulations partielles, il faut identifier les compromis entre plusieurs forces sur lesquels elles sont établies, dans un jeu à multiples acteurs portant des intérêts divers, permettant seul de saisir les facteurs de déstabilisation (et les crises qui peuvent être engendrées). Il est alors nécessaire de considérer la régulation des formes institutionnelles qui résulte d'un compromis 'politique' entre forces visant d'un côté à préserver l'autonomie des décisions d'acteurs situés dans un espace donné, de l'autre à exercer un contrôle, régulation qui est alors médiatisée par des formes institutionnelles stabilisées pour une durée plus ou moins longue. Pour être durable, ce compromis doit être ancré dans un registre de justification qui lui confère une légitimité nécessaire à l'engagement des parties, et mis en cohérence avec les autres éléments du 'système' au moyen des institutions. En tant que productrices importantes d'institutions, les politiques publiques déploient des répertoires dont les registres de justification permettent différentes formes idéales de cohérence. L'un de rôle des politiques publiques est de garantir un degré minimal de cohérence. La notion de cohérence sera saisie de cette manière comme une 'matière vivante', un état 'de stabilité relative et temporaire' en perpétuel ajustement dont l'un des élé- 
ments moteurs est le débat sur les formes de légitimité représentées par les différentes formes institutionnelles possibles. Le cadre théorique suivant cette perspective peut être esquissé par le rapprochement de trois programmes de recherche, dont l'intersection dessine ce que pourrait être l'analyse du fondement politique de la régulation sociale.

\section{LE FONDEMENT POLITIQUE DE LA RÉGULATION SOCIALE}

La variété persistante des modes de régulation met en scène différents types de compromis entre forces politiques, saisi dans des logiques de justification que les politiques publiques ont pour fonction de stabiliser. On définit trois sommets de l'analyse qui seront successivement étayés par l'analyse de la régulation sociale proposée par J.D. Reynaud, les travaux sur l'éthique du capitalisme de L. Boltanski, E. Chiappello et L. Thévenot et l'analyse « cognitives » des politiques publiques envisagée par B. Jobert et $\mathrm{P}$. Muller.

\section{La régulation comme art du compromis}

L'un des grands intérêts de l'analyse de la régulation sociale réalisée par J.-D. Reynaud est de considérer les dynamiques de négociations qui prennent le contrepied d'une hypothèse de cohérence et d'unité donnée a priori. Il est en effet possible, note l'auteur, de « Partir non de l'unité de la société, mais de la pluralité et de l'opposition des acteurs sociaux; non de l'effet unifiant de l'institution, mais du compromis symbolisé par le contrat ; non de l'hypothèse d'une conscience collective, foyer moral et religieux d'unité (ou, ce qui revient au même, d'un système de valeurs commun) mais d'une dispersion des intérêts, des préférences et des valeurs qui trouvent des points de 
rencontre et établissent ainsi des règles mutuellement admises; non d'une régulation générale de la société par les valeurs sociales, mais d'un ensemble, ni cohérent, ni continu, de régulations conjointes ponctuelles par des acteurs sociaux » [Reynaud, [1979], 1997, p. 112].

Reynaud se tient ainsi à équidistance de l'absence de régulation globale que de celle, inverse, de l'absence de régulation. Toutefois en propose-t-il une représentation plus complexe dans laquelle cette régulation est un produit conjoint résultant d'un jeu à plusieurs forces. Si l'on ne doit pas totalement abandonner l'idée d'une régulation cohérente, il faut en concevoir les termes réels comme le produit de deux forces différentes, de deux régulations mues par des logiques antagonistes - et co-produites par toute relation «qui s'établit entre un groupe et ceux qui veulent la régler de l'extérieur » [Reynaud, [1988], 1999, p. 162] : l'une recherchant une certaine autonomie, l'autre à des fins de contrôle.

Le résultat de cette confrontation est l'obtention d'une régulation réelle s'établissant sur un " compromis (souvent assez instable) entre autonomie et contrôle» [ibid., p. 167]. Néanmoins faut-il préciser comme le fait l'auteur que ce compromis n'est pas un point moyen, mais il définit un ensemble d'enjeux nécessairement plus important que les objectifs de chaque partie, dans lequel chacune d'elles néanmoins se retrouve : «Insistons cependant sur un point (car le terme de compromis peut induire une erreur) : le compromis en question n'est pas une moyenne entre des opinions ou des positions, ou un point intermédiaire; c'est une régulation, c'est-à-dire des règles générales, acceptables de part et d'autre, et constituant un ensemble raisonnablement cohérent » [ibid., p. 113]. 
Ce compromis est toutefois un équilibre plus ou moins stable du fait des tensions qui l'animent, des conflits qui s'y dénouent, ou des dénonciations critiques réalisées parfois par ses acteurs pour réaliser leurs fins ou tirer avantage de leur situation. Dès lors, il faut se demander pour quelles raisons les tensions pourront se résoudre (ou du moins être suspendues) autour d'un accord commun, permettant d'atteindre le niveau minimal de cohérence et d'efficacité permettant au « système » de se reproduire, au lieu de déboucher sur un conflit ou même, pourquoi pas, au chaos [Favereau, 2002]. Autrement dit, on doit s'intéresser aux formes de légitimité qui appuient ce compromis et dont la variété conduit à envisager différentes façons de prendre en compte la contrainte d'efficacité et la contrainte de cohérence (que vise notamment les politiques publiques) pour assurer la reproduction économique et sociale - tout en respectant la pluralité des choix des ménages.

\section{La régulation comme art de la justification}

Cette question est au cœur des analyses de Boltanski et Thévenot [1991] d'abord, et de Boltanski et Chiapello [1999] surtout, qui mettent en avant l'opération de justification permettant de parvenir à ce type de compromis. Ces derniers suggèrent que, pour fonctionner, se reproduire, ou se transformer, le capitalisme doit fournir une justification en termes de bien commun, par lequel les acteurs peuvent donner un sens à leurs actes, ou aux changements d'orientation qui leur sont imposés, mais qui en retour les contraint. Le capitalisme n'est donc pas seulement mû par les évolutions matérielles et les techniques de production, mais aussi et surtout par son Esprit - ce qui permet aux 
auteurs de retrouver les analyses de Weber et Hirschman. Deux éléments clé de leur analyse peuvent ici être soulignés :

D'une part, les auteurs soulignent que, pour être efficaces, les justifications doivent s'appuyer sur des espaces de calculs locaux, et des principes dont la portée est relativement générale, précisément pour être cohérent avec le fonctionnement économique et social global.

Dans ce cas, les agencements sociétaux, dans la mesure où ils sont soumis à un impératif de justification, tendent à incorporer la référence à un type de convention très générale orientée vers un bien commun et prétendant à une validité universelle - modélisée par les trois auteurs sous le concept de «cité ». Toutefois, étant donnée la pluralité des principes permettant d'établir une relation de bien commun, de tels arrangements ne pourront émerger comme solution stable que s'ils résistent aux épreuves de justification leur apportant une légitimité. Le résultat de cette opération est le plus souvent l'émergence d'un compromis, dont des grammaires variables sont reconstituées par Boltanski et Thévenot [1991], étendu par Boltanski et Chiapello [1999]1․ Se dégagent de cette perspective trois éléments nouveaux pour concevoir la régulation des comportements - et donc leur éventuelle cohérence.

Le premier concerne la possibilité d'identifier la logique profonde (sémantique) des différentes formes de régulation possibles, et de construire à partir d'elles des idéaux-types. Ainsi, la logique de la ré-

${ }^{1}$ Précisons que cet arrangement revêt un caractère « conventionnel » rendant compte du fait qu'il fait suite à un arbitrage - un choix politique - au sein d'une pluralité de représentations du bien commun [Batifoulier et Thévenon, 2001]. En outre, comme chez Reynaud, le compromis est compris non pas comme relâchement des contraintes de l'accord suscités par exemple par un abandon par les parties de leurs intérêts (une compromission), mais plutôt comme un renforcement de ces contraintes, les comportements devant se situer dans un cadre plus circonscrit. 
gulation - et des institutions qui lui donnent chair - est-elle avant tout fondée sur une cohérence sémantique : si institutions et comportements peuvent être agencés de façon cohérente, c'est parce qu'il y a, en amont même de la coordination de ces comportements et de l'adaptation des institutions, une coordination des jugements portés sur ces comportements et ces institutions. Cette coordination, c'est celle qui a lieu par l'épreuve de justification. La cohérence sociétale n'est donc plus abordée ici comme une simple complémentarité fonctionnelle, mais comme le résultat d'un jugement porté sur la légitimité des formes institutionnelles. Il est alors possible d'en déduire certaines hypothèses sur l'agencement des institutions, le registre de justification fournissant la « grammaire » nécessaire à leur mise en cohérence et la complémentarité qui peut être souhaitée.

Le second élément concerne la possibilité d'identifier plus clairement le rôle des institutions dans le processus de mise en cohérence et d'homogénéisation partielles des comportements, processus qui se construit par deux bouts, comme l'exprime très justement Favereau [2001] : «Le processus d'agrégation n'a donc rien d'une mécanique à sens unique. Il repose sur une inévitable tension entre l'ensemble des organisations qui fabriquent de la macroéconomie, et l'ensemble des institutions qui fabriquent de la microéconomie. La résultante de ces couples de forces antagonistes n'est certainement pas un social formant système, que le critère de jugement soit la coordination ou qu'il soit la reproduction. Le fait que le social ne fasse pas système va constituer dés lors, dans un monde caractérisé par les défauts de coordination et de reproduction, une justification en retour des institutions, qui, à défaut de mettre de l'ordre, cet ordre absolu rêvé par 
les langages de la coordination ou de la reproduction pures, tout au moins donnent du sens, ou plus exactement fournissent les clés sémantiques de la construction d'un 'monde commun'».

Enfin, la perspective proposée apporte des éléments complémentaires pour saisir la dynamique de changement des institutions. Trois concepts, maintenus dans une relation triangulaire, fournissent explicitement chez Boltanski et Chiapello [1999] les clés de cette dynamique :

- le concept de « cité », précédemment introduit, représente le premier sommet de ce triangle. Toute configuration sociétale renvoie en effet à deux niveaux logiques différents; celui des comportements ou actions possibles dans une configuration donnée; le second niveau considère des acteurs dotés d'un degré de réflexivité supérieur qui peuvent juger, au nom de principes universels, les actes contenus dans le premier ensemble. On se déplace donc vers l'étage des représentations, en rejoignant l'une des propositions centrales de l'analyse « conventionnaliste » des problèmes de coordination, selon laquelle : toute coordination des comportements suppose une coordination des jugements sur ces comportements, se faisant par le biais de représentations collectives; ces représentations comprennent alors une certaine idée du collectif, le concept de «cité » tirant cette «idée » vers la question de la justice [Bessis et al. dans ce volume ; ou Batifoulier et Thévenon, 2001]. Une telle représentation a alors pour fonction de légitimer et contraindre le domaine d'action des différents acteurs, et des politiques publiques en particulier. Les délibérations sur les formes de justice sont, dans cette perspective, un levier central du changement institutionnel. 
- Le concept « d'épreuve » légitime s'articule alors avec celui de délibération sur les principes de justice ; bien qu'inscrites dans des rapports de pouvoir, ces délibérations doivent, pour aboutir à un accord stable, satisfaire un impératif de justification en termes de bien commun $^{2}$. Le résultat fréquent de cette épreuve est l'établissement d'un compromis qui vise à satisfaire différents principes de justice. L'accord obtenu n'exclut en revanche aucunement la contestation, sous forme de critique ;

- La critique, fonctionne alors comme pivot de la dynamique institutionnelle et régulatrice; le rôle des critiques est d'autant plus important qu'elle peuvent s'exercer à deux étages : en premier lieu, la critique peut remettre en cause la pertinence des critères qui servent à l'évaluation d'une situation - sans toutefois contester ses fondements ; en ce sens, la critique peut servir à ajuster l'épreuve à la situation afin de mieux en évaluer le résultat, ceci allant dans le sens d'une « épuration » de l'épreuve pour la rendre plus identifiable³. Un ajustement des dispositifs institutionnels peut en dériver afin d'obtenir des résultats plus conformes aux effets attendus de ces dispositifs sur les comportements. Mais la critique peut s'exercer au niveau plus fondamental

${ }^{2}$ Les rapports de forces sont abordés ici comme $\mathrm{P}$. Muller le note à propos de l'action publique, qui soulignent que «les rapports de forces ne s'expriment pas par la simple confrontation «brute » des intérêts mais à travers des stratégies d'argumentation destinées à rendre légitimes les revendications - le recours à la force, compte tenu de son coût, étant réservé à des situations critiques qui ne peuvent être que l'exception » [Muller, 2005, p. 164].

${ }^{3}$ Il n'en reste pas moins que certains acteurs peuvent mettrent en place une stratégie de défense, visant à préserver leur autonomie, et réaliser pour cela certains « contournements » de la critique. Par des modifications des règles et des épreuves d'évaluation difficilement interprétables par la critique, les acteurs qui profitent de la pollution des règles ou de leur inefficacité au regard des critères affichés, peuvent chercher à maintenir le compromis dans un sens qui leur est favorable [Boltanski et Chiapello, 1999 ; Biencourt et al., 2001]. 
des principes et représentations qui fondent les évaluations. Cette distinction entre deux niveaux de critique appelle trois commentaires :

(i) d'une part, il importe, pour saisir la dynamique de changement institutionnel, de considérer avec attention les compromis établis entre différents intérêts à un niveau local, c'est-à-dire pour un domaine social ou une aire géographique particuliers, et « figés » dans les institutions. Ce compromis se lit dans le formatage particulier des dispositifs qui définit sa portée, ou la complémentarité et mise en relation avec les autres dispositifs de l'espace considéré ou des autres espaces. L'accord sur de tels compromis implique une tension pesant sur l'équilibre entre les différents principes ou intérêts représentés. La délibération sur les principes prévalant à la mise en place des dispositifs institutionnels ou à leur réforme peut alors conduire à modifier l'équilibre existant en privilégiant peu à peu l'un de ces principes. Se dessine ainsi un premier moteur de dynamique institutionnelle induite au niveau local, qui peut-être repérée par la façon dont se défont et se recomposent les compromis à travers les argumentaires mobilisés pour justifier les institutions et leur réformes ${ }^{4}$. De cette manière, il est possible de saisir l'évolution institutionnelle à ses niveaux syntaxique et sémantique, et d'être en mesure de mieux distinguer les différents processus d'évolution institutionnelle, repérés par Thélen [2003] selon trois grand types : (a) la conversion d'institutions, qui implique une

${ }^{4}$ Courtioux [2005] montre par exemple que la prime pour l'emploi en France modifie le cadre de justice locale en vigueur dans les politiques d'emploi, qui ouvre le chemin à une modification de la trajectoire institutionnelle. De la même manière, nous avions observé que l'extension de l'APE au rang 2 (puis au rang 1 avec la PAJE) porte atteinte le compromis (civique-domestique) sur lequel est fondée en France la régulation des relations emploi-famille qui vise à faciliter l'insertion dans l'emploi concomittante avec l'arrivée du premier enfant tout en «aidant » les sorties du marché du travail ayant lieu à un stade ultérieur [Thévenon, 2003]. 
redéfinition des objectifs et du contenu d'une institution en fonction du contexte et de la stratégie des acteurs, (b) la sédimentation, qui s'effectue par superposition de nouveaux arrangements qui suppléent les anciens), et enfin (c) l'hybridation des institutions existantes, sur la base de compromis forgés pour remplir un intérêts non prévu initialement.

(ii) Néanmoins la critique peut s'exercer à un niveau plus global qui vise l'imbrication globale de tous les éléments pouvant prétendre faire système. Et viser dans ce cas le manque de cohérence entre ces différents éléments, ou la nécessité de réformer de certains secteurs particuliers en cohérence avec un cadre global. Le cadrage ainsi réalisé vise à définir les vulnérabilités du système et à légitimer le «besoin » et le type de réformes. Les critiques portent alors sur le mode de régulation lui-même et mettent en avant l'incohérence qui peut exister entre le niveau de régulation globale et les politiques menées à un niveau plus local.

(iii) Enfin, on retrouve ici l'idée que l'analyse des régulations et de leur changement ne peut être menée qu'en considérant comment sont imbriqués les «trois I» que sont les Intérêts, les Idées, et les Institutions. Est traduite ici le fait partagé par différents néoinstitutionnalismes que tout comme les idées portent des intérêts, ces derniers n'ont pas de sens sans les idées et le format que leur confèrent les institutions. L'analyse du processus de changement doit alors considérer tout à la fois les conflits et compromis établis entre les intérêts concernés, le format imposé par les institutions héritées du passé, et la formulation du diagnostic et des solutions qui passe au travers de cadres cognitifs, normatifs et rhétorique [Palier et Surel, 2005]. 
L'analyse des «idées » est alors un élément clé du cadrage impliqué par tout processus de changement politique et institutionnel, la diffusion d'un discours plus ou moins unifié et visant différentes instances selon les pays étant lui-même du processus d'évolution institutionnelle [Schmidt, 2002].

Dans cette construction, les politiques publiques jouent un rôle à part dans la mesure un de leurs attributs est justement de créer (ou d'inciter à la création) d'institutions permettant de faire émerger $a d$ minima un degré suffisant de cohérence et de stabilité pour permettre aux acteurs de se coordonner et au système de se reproduire efficacement.

\section{La régulation comme art des politiques publiques}

Ce rôle apparaît tout à fait clairement dans l'analyse « cognitive » de l'action publique proposée par B. Jobert et P. Muller, qui voient dans toute politique publique un « processus de médiation sociale dans la mesure où l'objet de chaque politique publique est de prendre en charge les désajustements qui peuvent intervenir entre un secteur et la société globale» [Muller 2003, p. 24]. En souhaitant saisir ce processus, l'approche proposée est directement dynamique, pour répondre à notre question initiale sur la cohérence mais reformulée ici de la façon suivante : «comment 'produit-on' de l'ordre politique dans des sociétés de plu en plus complexes, de plus fragmentée et de plus en plus ouvertes sur l'extérieur » [Muller, 2000].

La décomposition que les auteurs proposent pour appréhender ce processus met en avant la dimension « cognitive» des politiques publiques puisque l'objet «n'est plus seulement de 'résoudre les pro- 
blèmes' mais de construire des cadres d'interprétation du monde» [Muller, 2000b]. Elaborer une politique publique revient, dans ce cas, à « construire une représentation, une image de la réalité sur laquelle on veut intervenir », en élaborant ainsi un référentiel [Muller, 2003, p. 42]. Ce référentiel est caractérisé par le contenu intellectuel et cognitif, et inclut une forme particulière de diagnostic et d'interprétation des problèmes. Il implique aussi certaines valeurs (i.e. des principes de légitimation de ce qui est ou ce qui devrait être) et des normes d'action qui définissent l'orientation générale de l'action publique (ce qu'il faut ou au contraire ne plus faire), et établit ainsi le rôle approprié de l'Etat et des instruments qu'il doit privilégier5. Le référentiel d'une politique est alors en réalité formé de deux composantes :

- un référentiel global qui est une « représentation générale autour de laquelle vont s'ordonner et se hiérarchiser les différentes représentations sectorielles. Il est formé d'un ensemble de valeurs fondamentales qui constituent les croyances de base d'une société, ainsi que d'une série de normes qui permettent de choisir entre des conduites ». Toutefois comme le précise Muller, "le référentiel global ne constitue pas une représentation parfaitement cohérente du monde. En effet, les valeurs qui le composent sont elles-mêmes enjeux de conflits $[\ldots]$ Le

${ }^{5}$ Ce référentiel est alors à la fois l'expression des contraintes structurelles et le résultat du travail sur le sens effectué par les acteurs [Muller, 2005]. En ce sens, il permet d'articuler l'effet contraignant des structures et les marges de jeu dont disposent les acteurs des politiques publiques. Dans cette perspective, le concept de référentiel constitue un outil pour penser (comme l'invitait J.-D. Reynaud) à la fois «l'autonomie des acteurs (parce que celle-ci s'exprime par le fait qu'ils «travaillent » le cadre cognitif et normatif dans lequel ils agissent en contribuant à rendre le réel à la fois plus intelligible et plus " désirable ») et les contraintes qui encadrent leur action (qui passent par le « sentiment d'inéluctabilité » du changement de ce cadre cognitif et normatif qui apparaît peu à peu comme une donnée irréductible, incontournable » [p. 188]. 
référentiel n'est pas un consensus mais il balise le champ intellectuel au sein duquel vont s'organiser les conflits sociaux » [p. 47] .

- un référentiel sectoriel, qui est une image du secteur. «Au sein d'un secteur donné coexistent toujours plusieurs conceptions de la nature et de l'extension des limites sectorielles, l'une d'entre elles étant en général dominante, souvent parce c'est elle qui est conforme à la hiérarchie globale des normes existant dans le référentiel global» [ibid., p. 48].

Le problème qui se pose alors à la constitution d'un référentiel politique est celui « de savoir comment ces différentes images de référence, dont l'articulation n'est pas toujours évidente, vont trouver un minimum de cohérence pour constituer un référentiel unifié » (p. 46). Il est résolu par « une opération d'encastrement du référentiel sectoriel dans le référentiel global qui est en quelque sorte l'acte fondateur d'une politique publique » [ibid., p. 49]. Cette opération implique une sélection d'informations et de modes opératoires. Mais elle implique aussi l'intégration de ces modes opératoires, «dans des valeurs qui vont leur donner la force et la désirabilité nécessaires ». Et Muller [2003] de nous prévenir : «Il ne faut pas se représenter le processus d'élaboration d'un référentiel comme un processus rationnel ou technique. En réalité, il s'agit d'un processus essentiellement culturel qui peut mettre en cause les valeurs les plus fondamentales des individus, ce qui explique d'ailleurs la violence qui accompagne parfois ces pha-

${ }^{6}$ En outre, comme le précise Muller [2005], le référentiel global constitue l'espace de sens qui va permettre de dépasser, jusqu'à un certain point, la situation «d'hyperchoix », c'est-à-dire de choix entre des options qui ne relèvent pas du même espace de sens, mais de logiques d'action qui sont par nature incommensurables. Le référentiel global permet de hiérarchiser ces options dans la mesure où «il délimite des valeurs, des normes et des relations causales qui s'imposent comme un cadre cognitif et normatif pour les acteurs engagés dans la confrontation de leurs intérêts » [p. 164]. 
ses de changement de la matrice de référence d'une politique » [p. 46]. Dès lors, l'analyse de l'action publique proposée est implicitement une théorie du changement, dès lors que survient un désajustement entre les différents sous-systèmes ou secteurs constituant la société [Muller, 2005].

Ainsi posé, le problème de l'articulation des représentations en un référentiel 'unifiant' renvoie de très près à la question de la justification évoquée précédemment, qui doit permettre la coordination des représentations visant les comportements développés dans un espace (ou un secteur) particulier, vis-à-vis de l'ensemble du système économique et social.

Il est naturel, dans cette perspective, de concevoir l'Etat en principal artisan de cette coordination, chargé d'arbitrer entre des conceptions concurrentes du bien commun - ce que met notamment en avant l'Economie des Conventions [voir notamment Salais, 1998 ; EymardDuvernay, 2001 ; Thévenon, 2001]. L'analyse « cognitive» des politiques publiques précédemment proposée donne à relire cette proposition, en considérant la tension qui s'exerce entre le pluralisme visé dans une société démocratique, et le souci de cohérence macroéconomique nécessaire à la reproduction du système. Dans une démocratie, si la garantie du pluralisme est bien l'une des prérogatives de l'Etat, les choix de politiques macroéconomiques impose aux politiques menées dans un secteur particulier certaines contraintes «d'ajustement », qui ne sont pas neutre vis-à-vis du «bien commun ». Aussi, si l'Etat est nécessairement une construction complexe, traversée par plusieurs principes de justice incommensurables, on ne peut concevoir, comme le souligne Eymard-Duvernay [2001], un Etat neutre à l'égard des 
conceptions du bien. La politique publique implique ainsi une évaluation particulière de ce qui constitue une action publique efficiente et un mode d'intervention particulier (Salais, 1998), dont dépend la mise en place de dispositifs institutionnels spécifiques pour obtenir des comportements compatibles avec un niveau minimal de cohérence.

L'approche «cognitive» des politiques publiques étaye ainsi l'idée que l'arbitrage politique implique une représentation de ce qui constitue une organisation économique et sociale «juste» (i.e. efficiente et équitable) - ce qui est désigné comme un référentiel global -, à partir de laquelle sont définies et hiérarchisées les attentes visant chacune de ses composantes. Il implique en outre une représentation de la configuration particulière de chaque espace - le référentiel sectoriel -, qui se dégage de façon dominante parmi la multitude des conceptions possibles, en raison de son adéquation au référentiel global, et donc aux principes de bien commun le fondant. La pluralité des principes de bien commun laisse néanmoins entrevoir une diversité de configurations possibles de représentations de l'efficience globale du système, et de la logique sectorielle correspondante. De plus, un certain désajustement entre les principes de bien commun émanant aux niveaux sectoriel et global laisse entrevoir une possibilité de situations de crise, et de dynamique d'ajustement? .

7 Néanmoins, comme le note Muller [2005], il est clair qu'en portant la réflexion à ce niveau de généralité, on ne vise pas à prévoir de manière précise les formes concrètes et le contenu spécifique du changement impliqué par telle politique : «c'est le sens du changement qui est prédictible et non ses modalités » [p. 170]. 


\section{CONCLUSION}

Résumons, pour conclure ce développement, ce que nous apprend le rapprochement des trois approches complémentaires proposé dans cette contribution :

On retient avec Reynaud que la régulation politique s'effectue selon un compromis, souvent implicite et assez instable, entre deux forces conflictuelles mais interdépendantes : l'une portée par les parties concernées visant un certain degré d'autonomie pour faire leurs choix - autonomie visant à respecter la pluralité des attentes et la variété des contextes ; et de l'autre, le contrôle réalisé notamment par l'Etat afin d'assurer une reproduction « juste » (i.e. efficace et équitable) du système économique et social, mais devant prendre en compte l'hétérogénéité des choix. La confrontation entre ces deux logiques peut être productrice de cohérence - et d'homogénéisation des comportements - mais celle-ci ne peut être que partielle. Dit autrement, une certaine hétérogénéité des comportements est toujours observable, mais la dispersion des comportements sera agencée de façon variable selon le type de compromis établi [Thévenon, 2003].

La réflexion est prolongée avec Jobert et Muller qui relèvent que toute action publique implique une opération de " mise en cohérence » d'une représentation globale devant assurer l'efficacité du système économique et social - i.e. l'efficacité de la politique macroéconomique - et d'une représentation sectorielle dominante renvoyant une image normative du secteur, et de la régulation partielle qui doit s'y opérer.

Enfin, le regard porté par l'Economie des Conventions sur cette opération d'assemblage au niveau des registres de justification impli- 
qué dans les choix politiques, et des évaluations de l'efficience variables qui en découlent. On met ainsi en avant la cohérence d'ordre sémantique qui fonde toute régulation, et dont dépend la mise en ordre «syntaxique » des institutions : la coordination des différents espaces nécessite préalablement une coordination des jugements visant les comportements et les institutions adoptés dans ces espaces. Cette coordination, c'est celle qui émerge par la justification des choix politiques qui opère un arbitrage entre différents principes pouvant prétendre au bien commun, et aboutit à des compromis. Cette épreuve de justification, inhérente au fonctionnement d'un régime démocratique, permet de mettre en cohérence les pratiques attendues dans un secteur particulier avec un fonctionnement global efficient de l'ensemble du système, au moins sous sa forme d'idéal-type. Cette justification entraîne un arbitrage en faveur de principes de « bien commun » particuliers dont dépend un découpage particulier des responsabilités de l'Etat et qui structurent de façon spécifique le mode d'intervention publique. La mobilisation d'un répertoire institutionnel particulier en découle, permettant de concevoir à la fois le formatage particulier de certains dispositifs institutionnel, et leur mise en relation particulière avec l'ensemble des autres dispositifs, conformément à une logique d'action publique donnée. L'analyse de ces dispositifs, et de leur agencement permet d'identifier les compromis institués. L'identification de principes suffisamment généraux pour être transposables d'un pays à l'autre permet de concevoir des idéaux-types auxquels les configurations observées dans les différents pays peuvent être comparées. 
Notons enfin que l'identification des compromis soutenant ces différences doit rendre intelligible les formes variables d'hétérogénéité des comportements observables d'un pays à l'autre. On vise ainsi à prendre en compte que les comportements se distinguent non seulement en moyenne d'un pays à l'autre, mais aussi en termes de dispersion, la variance des comportements étant agencée selon des caractères différents en fonction du compromis fondant la régulation. En explicitant les effets pouvant attendus sur les comportements de régulations typiques, l'analyse doit pouvoir se doter d'un instrument de vérification de ses hypothèses. En exhibant les principes de justification fondant les différentes formes de régulation, on a voulu montrer que l'évolution des formes de régulation n'est pas indépendantes de celles des représentations qui en assurent la légitimité. C'était un objectif d'établir ce lien, devant permettre de mieux appréhender les dynamiques de changement à l'œuvre au sein des institutions et des comportements qu'elles supportent.

\section{RÉFÉRENCES BIBLIOGRAPHIQUES}

Batifoulier P., ThÉVenon O. (2001), «Interprétation et fondement normatif des règles », in BATIFOULIER Ph. (Ed.), Théorie des Conventions, Paris, Economica, p. 219-252.

Berthelot, J.-M. (2000), «The implications of 'societal analysis' for a programme research ", in MAURICE M., SORGE A. (Eds), Embedding organizations, Amsterdam, John Benjamins Publishing Company, p. 89-99.

Biencourt O., Rebérioux A., Gabriel P. (2001), « La dynamique des conventions entre consensus et conflit», in BATIFOULIER PH. Théorie des Conventions, Paris, Economica, p. 253-277. 
Boltanski L., ThÉVenot L. (1991), De la Justification. Les Économies de la Grandeur, Paris, Gallimard.

Boltanski L., Chiapello E., (2000), Le nouvel esprit du capitalisme, Paris, Gallimard, coll. Nrf essai.

BOYER R., (2004), Une théorie du capitalisme est elle possible ?, Paris, Odile Jacob.

COURTIOUX P. (2005), « Dynamique institutionnelle et surdétermination de la justice locale ; quelles conséquences pour l'analyse des politiques publiques? », Colloque Conventions et Institutions, Paris, décembre 2003.

EYMARD-DUVERNAY F. (2001), «L'Économie des conventions a-t-elle une théorie politique ?», in BATIFOULIER Ph. (Ed.), Théorie des conventions, Paris, Economica, p. 279-297.

FAVEREAU O. (2002), « La théorie de la régulation sociale est-elle au centre de l'Economie des Conventions ? », in TERSSAC de G., (Ed.), La théorie de la régulation sociale de Jean-Daniel Reynaud, Presses Universitaires de France.

FAVereau O. (2001), «Les institutions et leur économie », Revue de Philosophie Economique, 4(2), p. 112-116.

HALl P., TAYlOR R., (1997), « La science politique et les trois néoinstitutionnalismes », Revue Française de Sciences Politiques, 47(4), p. 469-496.

MAURICE M. (2000), «The paradoxes of societal analysis. A review of the past and prospect for the future », in Maurice M., Sorge A. (Eds), Embedding organizations, Amsterdam, John Benjamins Publishing Company, p. 89-99.

Maurice M., Sellier F., Sylvestre J-J. (1982), Politique d'éducation et organisation industrielle en France et en Allemagne: essai d'analyse sociétale, Paris, Presses Universitaires de France, coll. Sociologies. 
Muller P. (2005), «Esquisse d'une théorie du changement dans l'action publique. Structures, acteurs et cadres cognitifs », Revue Française de Sciences Politiques, 55(1), Paris, p. 155-188.

Muller P. (2003), Les politiques publiques, coll. Que sais-je ?, Paris, Presses Universitaires de France, 4ème édition.

MULLER P. (2000), «L'analyse cognitive des politiques publiques, vers une sociologie politique de l'action publique », Revue française de science politique, vol. 50(2), p. 189-207.

PAlier B., Surel Y. (2005), « Les « trois I » et l'analyse de l'Etat en action », Revue Française de Sciences Politiques, 55(1), Paris, p. 7-32.

REYNAUD J.D. (1988), «Les régulations dans les organisations : régulation de contrôle et régulation autonome ", Revue française de sociologie, in REYNAUD J.D. (1997), Le conflit, la négociation et la règle, Editions Octarés, Toulouse, p. 157-158.

REYNAUD J.D. (1979), «Conflit et régulation sociale : esquisse d'une théorie de la régulation conjointe », Revue française de sociologie, in REYNAUD J.D. (1997), Le conflit, la négociation et la règle, Editions Octarés, Toulouse, p.109-118.

SALAIS R. (1998), « Action publique et conventions : état des lieux » in COMmaille J. et Jobert B. (Eds), Les métarmorphoses de la régulation politique, Paris, LGDJ, p. 55-81.

Thelen K. (2003), «Comment évoluent les institutions. Les enseignements d'une analyse historique comparative», L'Année de la régulation 2003-2004, 7, Presses de Sciences-Po, p. 13-43.

THÉRET B. (1997), «Méthodologie des comparaisons internationales, approches de l'effet sociétal et de la régulation : fondements pour une lecture structuraliste des systèmes nationaux de protection sociale », L'Année de la Régulation, 1, p. 163-228.

THÉVENON O. (2003), Les relations emploi-famille en Europe. Fondement socio-économique des comportements féminins en Allemagne, France, 
Espagne, aux Pays-Bas et Royaume-Uni, Thèse de doctorat en Sciences économiques, Université Paris X-Nanterre. 